\title{
Predicting Gleason sum upgrading from biopsy to radical prostatectomy pathology: a new nomogram and its internal validation
}

\author{
Xiaochuan Wang ${ }^{\dagger}$, Yu Zhang ${ }^{\dagger}$, Fengbo Zhang, Zhengguo Ji, Peiqian Yang and Ye Tian ${ }^{*}$
}

\begin{abstract}
Background: To explore the rate of Gleason sum upgrading (GSU) from biopsy to radical prostatectomy pathology and to develop a nomogram for predicting the probability of GSU in a Chinese cohort.

Methods: We retrospectively reviewed our prospectively maintained prostate cancer (PCa) database from October 2012 to April 2020. 198 patients who met the criteria were enrolled. Multivariable logistic regression analysis was performed to determine the predictors. Nomogram was constructed based on independent predictors. The receiver operating curve was undertaken to estimate the discrimination. Calibration curve was used to assess the concordance between predictive probabilities and true risks.

Results: The rate of GSU was $41.4 \%$, whilst GS concordance rate was $44.4 \%$. The independent predictors are prostate specific antigen (PSA), greatest percentage of cancer (GPC), clinical T-stage and Prostate Imaging Reporting and Data System (PI-RADS) score. Our model showed good discrimination (AUC of 0.735). Our model was validated internally with good calibration with bias-corrected C-index of 0.726 .

Conclusions: Utilization of basic clinical variables (PSA and T-stage) combined with imaging variable (PI-RADS) and pathological variable (GPC) could improve performance in predicting actual probabilities of GSU in the 24-core biopsy scheme. Our nomogram could help to assess the true risk and make optimal treatment decisions for PCa patients.
\end{abstract}

Keywords: Prostatic neoplasms, Neoplasm grading, Biopsy, needle, Prostatectomy, Nomograms

\section{Background}

Gleason score is a critical factor for both risk stratification and disease management in prostate cancer $(\mathrm{PCa})$. However, it was reported that the concordance of Gleason score between initial biopsy and final radical prostatectomy (RP) pathology was barely satisfactory [1]. Gleason sum upgrading (GSU) may affect assessment of the true PCa risk and treatment options for PCa patients. For instance, active surveillance is recommended for

\footnotetext{
*Correspondence: tianye166@126.com

${ }^{+}$Xiaochuan Wang and Yu Zhang have contributed to the study consistently

Department of Urology, Capital Medical University Affiliated Beijing

Friendship Hospital, No. 95, Yongan Road, Xicheng District, Beijing,

People's Republic of China
}

patients with Gleason 6 or $3+4$ but not appropriate for patients with Gleason $4+3$ or above [2]. Patients with organ-confined tumors of Gleason 8 or above should underwent RP followed by lymph node dissection and other ancillary therapies in case of PSA failure [3]. Similarly, external beam radiation therapy is recommended to be combined with androgen deprivation therapy in patients with Gleason $4+3$ or above [4]. It has been demonstrated in large-scale studies that patients with GSU were significantly associated with biochemical recurrence, distant metastasis and death from $\mathrm{PCa}[5,6]$.

To address this matter, our study aimed to examine the rate of GSU between biopsy and final pathology and determine independent predictors for GSU. Moreover, our study developed a nomogram including clinical, 
imaging and pathologic variables based on Chinese population. We therefore expect to help reassess the risk after biopsy and select optimal treatment modalities for PCa patients after a comprehensive evaluation.

\section{Methods}

\section{Data acquisition and patient selection}

From our prospectively maintained institutional review board-approved prostate biopsy database, we identified 2039 consecutive men who underwent biopsy at our institution (1st October 2012 to 30th April 2020). 212 patients who underwent RP at our institution met the inclusion criteria. The exclusion criteria were as follows: (1) patients treated with neoadjuvant therapy before RP; (2) patients with missing data. Finally, 198 eligible patients entered into our study cohort. Two researchers independently browsed the database, extracted the data and cross-checked. This study did not contain any human participants or animals and it received ethics approval from Capital Medical University affiliated Beijing Friendship Hospital Ethics Committee for database access (2019-P2-081-01).

\section{Clinical evaluation}

Prostate volume (PV) was calculated using anterior-posterior diameter, superior-inferior diameter and left-right diameter which were obtained from multiparametric magnetic resonance imaging (mpMRI). Prostate specific antigen (PSA) was measured before digital rectal examination (DRE) and biopsy. Clinical T-stage was assigned according to the 2017 TNM system which relied on findings of biopsy, DRE and mpMRI.

As recommended [7], the prostate mpMRI was performed before biopsy at a 3-T $(\mathrm{T})$ system. MpMRI protocol consisted of T2-weighted imaging (T2WI), diffusion-weighted imaging (DWI) with apparent diffusion coefficient map (ADC), and dynamic contrast-enhanced (DCE) sequences and calculated $b$ value of 1000 or above. Urological radiologists who were experienced with PIRADS score and were blinded to pathology as well as clinical data evaluated all the images and performed scoring.

\section{Biopsy and pathological evaluation}

Patients received 18-gauge transperineal needle biopsy under general anesthesia in a dorsal lithotomy position. The biopsy was performed under the guidance of transrectal ultrasonography (TRUS) utilizing an 24-core extended scheme. There were twenty needles in the peripheral zone (PZ) from apex and mid to posterior base and four needles in the transitional zone (TZ). Each core was submitted in a separate container. 24-core systematic biopsy was a standard practice across our center performed by experienced urologists. Gleason sum was assigned core by core by a specialized team of urogenital pathologists using global Gleason score. All RP specimens were examined for prostatectomy Gleason sum by the same team. Hence, non-uniform interpretation of reports between pathologists and clinicians could be avoided [8]. Tertiary patterns were included in biopsy, but not included in prostatectomy specimens. GSU was defined as any Gleason sum upgrading from biopsy to RP. GPC (greatest percentage of cancer in a single core) and fraction of positive cores (FPC) were applied to measure tissue tumor extent (TTE) in biopsy cores [9].

\section{Statistical analysis}

The patients were divided into two groups as those with GSU and those without GSU. Age, metabolic status, body mass index (BMI), interval from biopsy to RP, PV, PSA, PSA density (PSAD), clinical T-stage, PI-RADS score and biopsy specimen features were analyzed in all patients. Normality of distribution of the variables was checked using the Shapiro-Wilk tests and P-P plots. Normally distributed numerical variables were analyzed by the student $t$ tests. Mann-Whitney $\mathrm{U}$ tests were applied to determine the significance of nonnormally distributed numerical variables. Chi-square tests were used for categorical variables. Univariable regression analysis was performed followed by the multivariable analysis. Variables that were found statistically significant in univariable analysis entered the multivariable analysis in a forward stepwise selection (probability of 0.05 ). The nomogram was constructed with validated independent predictors. The performance of the prediction model was evaluated from the aspect of discrimination and calibration. Discrimination was measured using the receiver operating curve (ROC) with the area under the curve (AUC) value. Calibration was assessed by visually inspecting the plots of predicted probability and actual probability. Bias-corrected C-index was also calculated to be compared with original C-index (AUC value). Internal validation was performed by bootstrap resampling $(n=1000)$ to evaluate the accuracy estimates and to reduce overfit bias. Tests were 2 sided and $P<0.05$ was the threshold for statistical significance. Statistical tests were performed using computer software of SPSS version 24.0 and R version 4.0.2.

\section{Results}

A statistical significance was met when it came to variables: cT-stage, PI-RADS score, PSA and GPC (all $P<0.05)$. Patients in GSU subgroup had a higher ratio for $\mathrm{T}_{2 \mathrm{~b}-2 \mathrm{c}}$ cancers $(66.3 \%)$ and PI-RADS score of 4-5 (89.9\%), higher PSA level $(15.7 \mathrm{ng} / \mathrm{ml})$ and higher GPC (90.0\%). Other demographic details were shown in Table 1. 
Table 1 Demographics of patients underwent biopsy followed by prostatectomy in total and subgroups

\begin{tabular}{|c|c|c|c|c|}
\hline Variables & Total $(n=198)$ & GSU $(n=89)$ & Non-GSU $(n=109)$ & $P$ value \\
\hline Age at biopsy (year), M(IQR) & $67.0(63.0-71.0)$ & $67.0(62.0-71.0)$ & $67.0(63.0-71.0)$ & $0.496^{a}$ \\
\hline MS (n, \%) & - & - & - & 0.150 \\
\hline Absence & 156(78.8) & $66(74.2)$ & $90(82.6)$ & - \\
\hline Presence & $42(21.2)$ & $23(25.8)$ & $19(17.4)$ & - \\
\hline BMI, M(IQR) (kg/m²) & $24.7(22.9-26.9)$ & $25.0(23.1-27.2)$ & $24.6(22.3-26.7)$ & $0.345^{\mathrm{a}}$ \\
\hline Interval from biopsy to RP, M(IQR) (d) & $21.0(14.0-34.3)$ & $21.0(14.0-31.5)$ & $21.0(14.0-35.0)$ & $0.771^{b}$ \\
\hline PV, M(IQR) (ml) & $39.0(28.1-53.0)$ & $38.8(28.7-52.8)$ & $39.2(23.9-53.4)$ & $0.801^{b}$ \\
\hline Pre-biopsy PSA level, M(IQR) (ng/ml) & $14.2(8.6-28.7)$ & $15.7(9.1-35.9)$ & $13.1(7.8-23.6)$ & $0.032^{b}$ \\
\hline Pre-biopsy PSAD, M(IQR) (ng/ml²) & $0.37(0.21-0.79)$ & $0.46(0.23-1.01)$ & $0.34(0.20-0.74)$ & $0.061^{b}$ \\
\hline Clinical T-stage $(n, \%)$ & - & - & - & 0.002 \\
\hline$T_{1 c-2 a}$ & 63(31.8) & $18(20.2)$ & $45(41.3)$ & $0.001^{c}$ \\
\hline$T_{2 b-2 c}$ & 105(53.0) & $59(66.3)$ & $46(42.2)$ & $0.117^{d}$ \\
\hline$T_{3 a-3 b}$ & $30(15.2)$ & $12(13.5)$ & $18(16.5)$ & $0.270^{e}$ \\
\hline PI-RADS score (n, \%) & - & - & - & $<0.001$ \\
\hline $2-3$ & $51(25.8)$ & $9(10.1)$ & $42(38.5)$ & - \\
\hline $4-5$ & $147(74.2)$ & $80(89.9)$ & $67(61.5)$ & - \\
\hline Cores obtained (n, \%) & - & - & - & 0.480 \\
\hline $12-23$ & $16(8.1)$ & $9(10.1)$ & $7(6.4)$ & - \\
\hline 24 & 165(83.3) & 74(83.1) & $91(83.5)$ & - \\
\hline $25-30$ & $17(8.6)$ & $6(6.7)$ & $11(10.1)$ & - \\
\hline FPC, M(IQR) (\%) & $25.0(13.0-42.0)$ & $25.0(12.5-48.0)$ & $25.0(13.0-42.0)$ & $0.906^{b}$ \\
\hline GPC, M(IQR) (\%) & $70.0(30.0-90.0)$ & $90.0(50.0-90.0)$ & $50.0(30.0-90.0)$ & $0.001^{b}$ \\
\hline Experience in biopsy $(n, \%)$ & - & - & - & 0.886 \\
\hline Senior & 79(39.9) & $36(40.4)$ & $43(39.4)$ & - \\
\hline Junior & $119(60.1)$ & $53(59.6)$ & $66(60.6)$ & - \\
\hline
\end{tabular}

GSU Gleason sum upgrade, $M$ median, IQR interquartile range, $M S$ metabolic syndrome, $R P$ radical prostatectomy, $B M I$ body mass index, $P V$ prostate volume, $P S A$ prostate specific antigen, PSAD PSA density, PI-RADS prostate Imaging Reporting and Data System, FPC fraction of positive cores, GPC the greatest percentage of cancer

a Analyzed by the student $t$ test

b Analyzed by the Mann-Whitney $U$ test

c Comparison between $\mathrm{T}_{1 \mathrm{c}-2 \mathrm{a}}$ and $\mathrm{T}_{2 \mathrm{~b}-2 \mathrm{c}}$

${ }^{d}$ Comparison between $\mathrm{T}_{2 \mathrm{~b}-2 \mathrm{c}}$ and $\mathrm{T}_{3 \mathrm{a}-3 \mathrm{~b}}$

e Comparison between $\mathrm{T}_{1 \mathrm{c}-2 \mathrm{a}}$ and $\mathrm{T}_{3 \mathrm{a}-3 \mathrm{~b}}$

Table 2 Distribution in Gleason scores between the biopsy cores and RP specimens

\begin{tabular}{|c|c|c|c|c|c|c|c|}
\hline \multirow[t]{2}{*}{ Biopsy Gleason sum } & \multicolumn{7}{|c|}{ RP Gleason sum } \\
\hline & 6 & $3+4$ & $4+3$ & 8 & 9 & 10 & Total \\
\hline 6 & 28 & 28 & 4 & 6 & 3 & & 69 \\
\hline $3+4$ & 3 & 24 & 9 & 13 & 4 & & 53 \\
\hline $4+3$ & 1 & 7 & 13 & 10 & 1 & & 32 \\
\hline 8 & & 2 & 9 & 7 & 9 & & 27 \\
\hline 9 & & 1 & 2 & & 9 & 1 & 13 \\
\hline 10 & & & & 1 & 2 & 1 & 4 \\
\hline Total & 32 & 62 & 37 & 37 & 28 & 2 & 198 \\
\hline
\end{tabular}


We found that concordance rate between initial biopsy pathology and final RP specimens was $41.4 \%$ (82/198), whilst GSU was $44.4 \%$ (88/198). Downgrading was found in $14.1 \%(28 / 198)$ of patients (Table 2). Our study further divided the entire cohort into three groups according to the biopsy Gleason sum $(6,7, \geq 8)$. The percentage of GSU was highest in patients with Gleason sum 6, while the concordance rate was highest in patients with Gleason sum 7. The percentage of downgrading was highest in patients with Gleason sum 8-10 (Fig. 1).

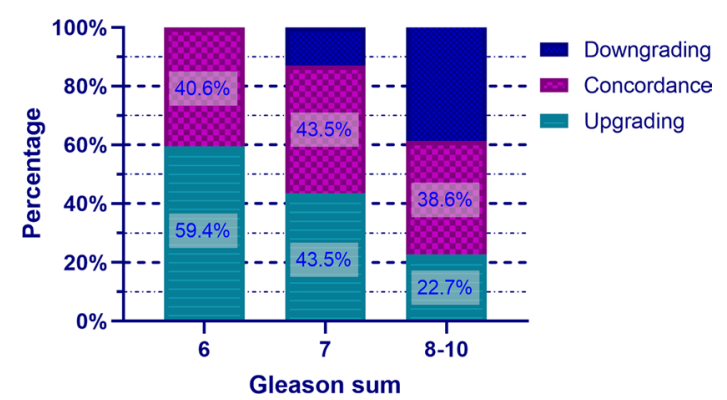

Fig. 1 Stacked bar chart of the distribution and magnitude of Gleason sum change from biopsy to prostatectomy pathology in three cohorts of men with different Gleason sum
Univariate analysis revealed that PSA, PSAD, GPC, cT-stage and PI-RADS score were the predictors of GSU (all $P<0.05$ ). Multivariate analysis confirmed that PSA, GPC, cT-stage and PI-RADS score were independent predictors of GSU (all $P<0.05$ ) (Table 3). Additionally, we managed to find whether there were predictors for downgrading and consequently no significant factor was found in regression analysis. The nomogram was constructed based on four predictors of multivariable analysis. The AUC of GPC, PSA, PI-RADS score and cT-stage were $0.636,0.589,0.642$ and 0.579 respectively. The AUC of GSU model was 0.735 , which showed good performance of discrimination (Figs. 2, 3).

Our internally validated calibration plot demonstrated virtually ideal predictions that the rate of predicted probabilities closely paralleled the observed rate (nearly corresponded to the $45^{\circ}$ line) with bias-corrected C-index of 0.726 (Fig. 4). Our model predicting GSU might underestimate the risk at the probability range of $53-64 \%$ and below $41 \%$. The overestimate risk was at the range of $42-52 \%$ and above $64 \%$.

\section{Discussion}

A systematic review including 14,839 patients reported that concordance rate was $63 \%$, while overall upgrading was found in 30\% [1]. Based on British Association

Table 3 Predictors of Gleason sum upgrading from biopsy to RP

\begin{tabular}{|c|c|c|c|c|}
\hline \multirow[t]{2}{*}{ Variables } & \multicolumn{2}{|c|}{ Univariable analysis } & \multicolumn{2}{|c|}{ Multivariable analysis } \\
\hline & OR $(95 \% \mathrm{CI})$ & $P$ value & OR $(95 \% \mathrm{Cl})$ & $P$ value \\
\hline Age at biopsy & $0.98(0.94-1.03)$ & 0.494 & - & - \\
\hline MS, presence vs absence & $1.65(0.83-3.28)$ & 0.152 & - & - \\
\hline BMI & $1.05(0.95-1.15)$ & 0.343 & - & - \\
\hline Interval from biopsy to RP & $1.00(0.99-1.00)$ & 0.253 & - & - \\
\hline PV & $1.00(0.99-1.01)$ & 0.713 & - & - \\
\hline Pre-biopsy PSA level & $1.01(1.00-1.02)$ & 0.009 & $1.02(1.00-1.03)$ & 0.007 \\
\hline Pre-biopsy PSAD & $1.42(1.01-2.01)$ & 0.044 & $0.41(0.14-1.12)$ & 0.522 \\
\hline Clinical T-stage & - & - & - & - \\
\hline T1c-2a & 1.00(Reference) & - & 1.00(Reference) & - \\
\hline$T 2 b-2 c$ & $3.21(1.64-6.26)$ & 0.001 & $4.13(1.82-7.24)$ & 0.042 \\
\hline T3a-3b & $1.67(0.67-4.15)$ & 0.272 & $2.39(0.86-4.85)$ & 0.122 \\
\hline PI-RADS score $4-5$ versus $2-3$ & $5.57(2.53-12.27)$ & $<0.001$ & $4.81(2.07-11.18)$ & $<0.001$ \\
\hline Cores obtained & - & - & - & - \\
\hline $12-23$ & 1.00(Reference) & - & - & - \\
\hline 24 & $0.63(0.23-1.78)$ & 0.385 & - & - \\
\hline $25-30$ & $0.42(0.10-1.72)$ & 0.231 & - & - \\
\hline FPC & $1.24(0.37-4.14)$ & 0.731 & - & - \\
\hline GPC & $5.34(1.98-14.39)$ & 0.001 & $3.09(1.05-9.07)$ & 0.040 \\
\hline Experience in biopsy, senior versus junior & $1.04(0.59-1.85)$ & 0.886 & - & - \\
\hline
\end{tabular}

$O R$ odds ratio, $C l$ confidence interval, $M S$ metabolic syndrome, $R P$ radical prostatectomy, $B M I$ body mass index, $P V$ prostate volume, $P S A$ prostate specific antigen, $P S A D$ PSA density, PI-RADS Prostate Imaging Reporting and Data System, FPC fraction of positive cores, GPC the greatest percentage of cancer 


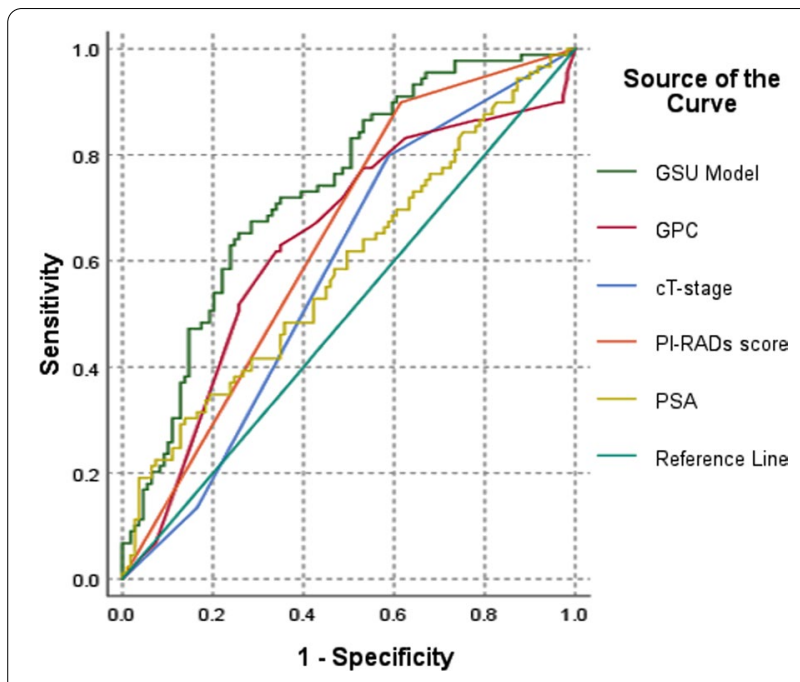

Fig. 2 ROC of Gleason sum upgrading model and independent predictors. GSU Gleason sum upgrading, GPC the greatest percentage of cancer, PSA prostate specific antigen, PI-RADS Prostate Imaging Reporting and Data System of Urological Surgeons (BAUS) Radical Prostatectomy Registry database, a study of 17,598 patients [10] demonstrated that concordance rate was $58.9 \%$, whilst upgrading rate was $25.5 \%$. Our study showed that the concordance rate was $41.4 \%$, while GSU was $44.4 \%$. Concordance rates and upgrading rates vary from study to study which might be attributed to reasons as follows: different demographic characteristics of study cohorts, sampling error of biopsy approach, different biopsy techniques, variability of pathologic assignment and non-uniform interpretation of pathology reports.

PSA is a widely used indicator for both risk stratification and prognosis evaluation, and it also plays a role in predicting Gleason sum upgrading [11-19]. High level of serum PSA was partially attributed to large prostate glands in elder patients. However, Davis et al. [18] found that PSA was still an independent predictor after adjusting for PV. In the current study, we found that PSA was also predictive and increasing PSA level was strongly correlated with GSU. For every increasing PSA unit, there was a $2 \%$ increased risk of upgrading. Hence, urologists

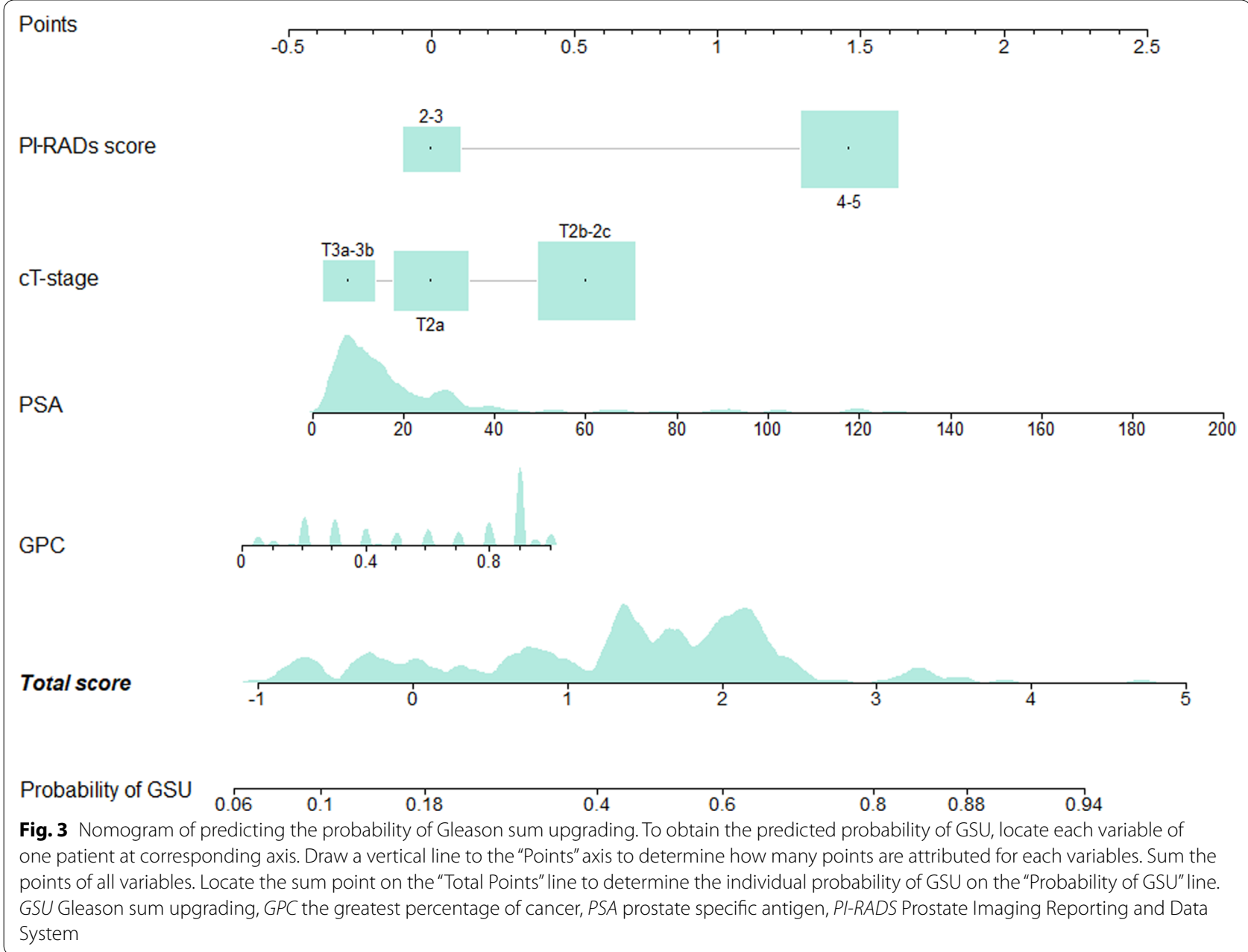




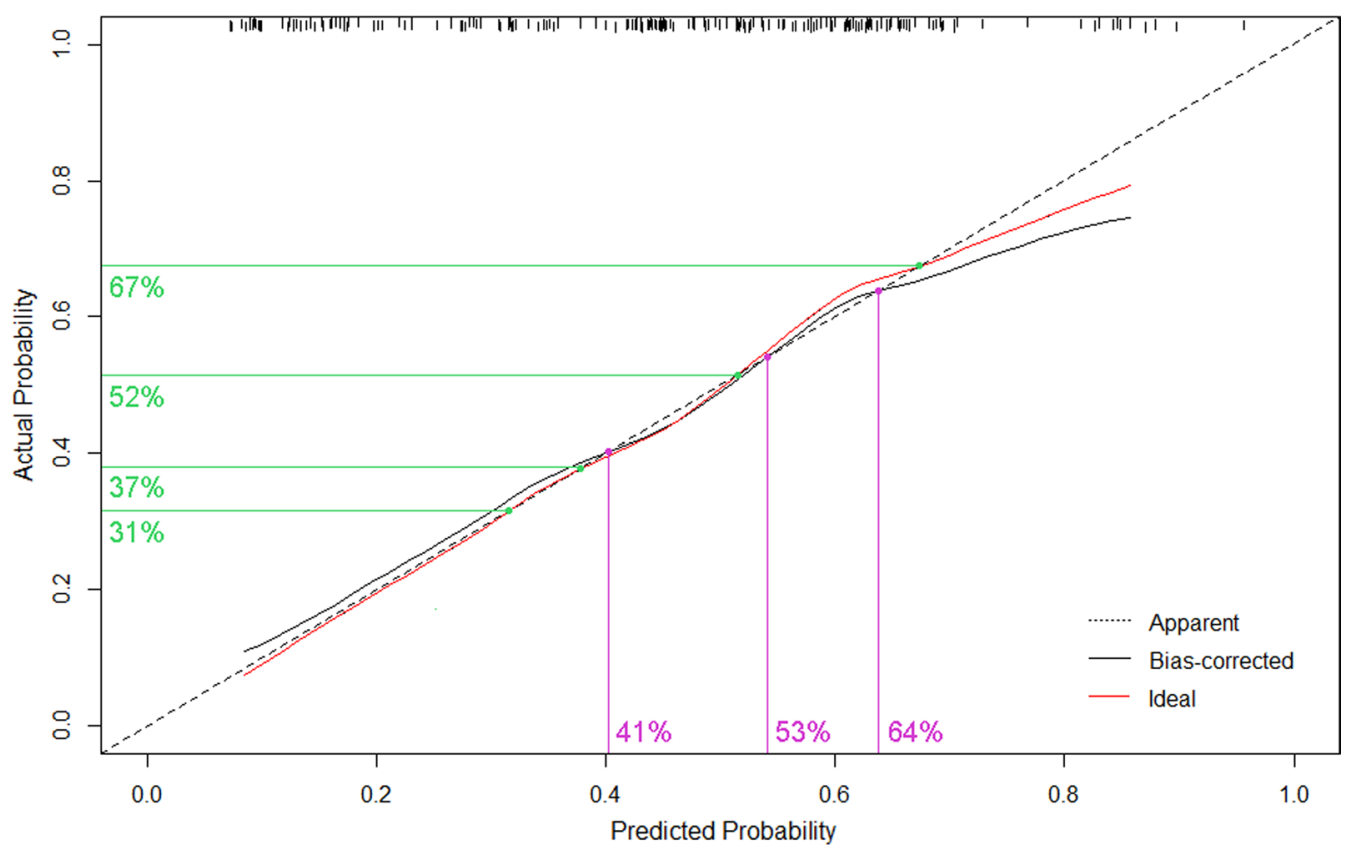

Fig. 4 Calibration curve of the Gleason sum upgrading model. The nomogram-predicted probabilities are comparable to the actual probabilities of GSU. Perfect prediction would correspond to a slope of 1 (diagonal $45^{\circ}$ broken line). The solid red line indicates the original predictive ability. The solid black line indicates the bootstrap-corrected model performance

should raise concern about the cumulative effect in patients with high level of PSA.

Clinical T-stage is another important preoperative indicator as for guiding treatment decisions and evaluating prognosis, and it was also found to be predictive of upgrading in several studies [11, 12, 15-17]. Advanced $\mathrm{T}$-stage is in general correlated with large tumor volume and sampling error trends to appear when biopsies are performed in patients with large tumor focus. However, $\mathrm{T}_{2}$ tumors were associated with more upgrading compared to $\mathrm{T}_{3}$ tumors in the study of Chun et al. [12], the finding of which was consistent with the current study. $\mathrm{PCa}$ occurs mainly in the PZ which is compressed by hyperplastic TZ tissue in elder patients. Under this circumstance, small tumor volume and tumor extracapsular extension might coexist. Additionally, diagnostic accuracy of predicting RP Gleason sum varied in different prostate zones which might give another explanation to this phenomenon [20]. However, the correlation between GSU and clinical T-stage needs further investigations.

GPC which was commonly used by pathologists to determine TTE was reported to be correlated with upgrading [13, 18, 19, 21]. In the current study, this pathologic variable was an independent predictor of GSU. High maximum percentage of cancer reflects larger tumor volume which might increase sampling error. Under this circumstance, high-grade masses in large tumor foci would be less detected. Even if small GPC provides pathologists with inadequate information which could increase the possibilities of incorrect scoring, it seems that impacts on the assignment of primary and secondary patterns are slight.

PI-RADS score was validated to have good performance for both PCa detection and foci localization [22]. The utilization of mpMRI was confirmed the value of differentiating clinically significant $\mathrm{PCa}$ from indolent tumors [23]. We found that PI-RADS score was also an independent predictor of GSU, which is consistent with the findings of Song et al. [24]. Gleason sum were more likely to be upgraded among patients with PI-RADS 4-5 (54.4\% compared with $17.6 \%$ for PI-RADS 2-3). Highgrade patterns with MRI manifestation of PI-RADS 4-5 which are not identified in biopsy cores result in GSU. Gondo et al. [25] found that Gleason sum downgrading was associated with negative MRI findings, which demonstrated that mpMRI might have favorable diagnostic performance in predicting Gleason sum change.

We successfully developed and validated a model predicting Gleason sum upgrading from biopsy to RP utilizing clinical variables (PSA and T-stage) combined with imaging variable (PI-RADS) and pathological variable (GPC). Our model is $73.5 \%$ accurate and its predictions closely approximate the observed rate. Our study was not the first one to construct models or nomograms. 
Chun et al. [12] built a model including PSA, clinical stage and biopsy Gleason score and yielded accuracy of 0.804 based on a large scale of population. The number of biopsy cores ranged from 6 to 12 in their study. However, excluding the number of cores from multivariable analysis would affect the stability of their model. Moussa et al. [16] incorporated manifold variables especially pathologic parameters into their model. However, including statistically insignificant variables leaded to their model becoming unstable. Therefore, their model yielded a C-index of 0.68. Both Kulkarni et al. [14] and Capitanio et al. [26] have built models for patients with Gleason sum 6, which yielded accuracy of 0.71 and 0.66 respectively. These two models fit low-risk population well, however, which could not represent the real situation of overall GSU. Both Wang et al. [17] and He et al. [27] developed models based on Chinese population. The model of Wang et al. [17] included only PSA, clinical stage and biopsy Gleason score which showed favorable statistical performance with C-index of 0.795 . Wang et al. also externally validated Chun's model in the set of Chinese population and the results showed poor concordance between the predicted and observed probabilities. What Wang et al. have found demonstrated that new models need to be constructed to fit specific population and should be further validated with large patient cohorts. He et al. [27] built models predicting upgrading for both overall patients and patients with $G S=6$. Two models including variables such as BMI and FPC showed good performance and yielded the AUC of 0.753 and 0.727 respectively. Our model incorporated not only basic clinical features such as PSA and clinical stage, but also pathologic and imaging parameters, which is more suitable for clinical practice. Including independent predictors leaded to our model becoming more stable and the predicted probabilities closely approximated to the actual risk in calibration plot. When probabilities ranged from 53 to $64 \%$ and below $41 \%$, underestimation of the risk occurred, while overestimation of risk ranged from 42 to $52 \%$ and above $64 \%$.

According to our present study, several clinical implications might be taken into consideration. Patients who have low-risk PCa but high probabilities of GSU could consider curative therapies instead of active surveillance in case of delayed treatment. For these patients who are under active surveillance could adhere to more active follow-up policies. On the contrary, patients with low probabilities of GSU who are unwilling to receive aggressive treatment or have contraindications of operation or radiotherapy are more confidence to undergo the active surveillance. According to the probabilities of GSU, extended periods of close clinical follow-up could be warranted. Moreover, patients might benefit from resection of neurovascular bundle or lymph node dissection who are at high risk of GSU. Similarly, the hormonal therapy as ancillary treatment to radiotherapy might be considered in patients with high probabilities of GSU. These clinical recommendations might give urologists more confidence in clinical decision-making. New model might provide urologists not only precise and comprehensive assessment of $\mathrm{PCa}$ risk but also personalized and optimal treatment options for PCa patients. However, there is still far to go before robust evidence emerges.

There are limitations of the present study. First of all, information from database included potential inaccuracy due to the retrospective nature. Secondly, our study was lack of large population because of our strict indications to perform RP and monocentric design. Thus, we need further prospective large-scale studies with selective patients for validation. Thirdly, external validation for our model would be furtherly necessitated, even if our model served as a statistically well-performed tool. Fourthly, only patients undergone RP were selected into cohort which might not represent the reality. Finally, the accuracy of our model could potentially be improved by integrating findings of genetic and molecular biomarker analysis.

\section{Conclusions}

Utilization of basic clinical variables (PSA and T-stage) combined with imaging variable (PI-RADS) and pathological variable (GPC) could improve performance in predicting actual probabilities of GSU in the 24-core biopsy scheme. Our nomogram could help to assess the true risk and make optimal treatment decisions for PCa patients. However, our nomogram needs to be tested for the performance in an external dataset or other study cohorts.

\section{Abbreviations}

GSU: Gleason sum upgrading; PCa: Prostate cancer; RP: Radical prostatectomy; MS: Metabolic syndrome; BMI: Body mass index; PV: Prostate volume; PSA: Prostate specific antigen; PSAD: PSA density; PI-RADS: Prostate Imaging Reporting and Data System; mpMRI: Multiparametric magnetic resonance imaging; GSU: Gleason sum upgrade; GPC: Greatest percentage of cancer; FPC: Fraction of positive cores; TTE: Tissue tumor extent; ROC: Receiver operating curve; AUC: Area under the curve.

\section{Acknowledgements}

Not applicable.

\section{Authors' contributions}

$X W$ was a major contributor in conception and writing the manuscript. XW and $Y Z$ analyzed and interpreted the patient data. ZJ and FZ drafted and supervised the work and PY and YT substantively revised it. All authors read and approved the final manuscript and have agreed both to be personally accountable for the author's own contributions and to ensure that questions related to the accuracy or integrity of any part of the work, even ones in which the author was not personally involved, are appropriately investigated, resolved, and the resolution documented in the literature. All authors read and approved the final manuscript. 


\section{Funding}

Not applicable.

\section{Availability of data and materials}

The datasets used and/or analysed during the current study are available from the corresponding author on reasonable request.

\section{Ethics approval and consent to participate}

This study did not contain any human participants or animals. Our study was approved by the Ethics Committee of Capital Medical University affiliated Beijing Friendship Hospital, Beijing, China. The Ethics Committee provided the permission to access the prostate biopsy database. The ethical approval number is 2019-P2-081-01.

\section{Consent for publication}

Not applicable.

\section{Competing interests}

The authors declare that they have no competing interests.

Received: 29 June 2020 Accepted: 15 December 2020

Published online: 06 January 2021

\section{References}

1. Cohen MS, Hanley RS, Kurteva T, et al. Comparing the Gleason prostate biopsy and Gleason prostatectomy grading system: the Lahey Clinic Medical Center experience and an international meta-analysis. Eur Urol. 2008:54:371-81.

2. Thomsen FB, Brasso K, Klotz LH, et al. Active surveillance for clinically localized prostate cancer-a systematic review. J Surg Oncol. 2014;109:830-5.

3. Briganti A, Larcher A, Abdollah F, et al. Updated nomogram predicting lymph node invasion in patients with prostate cancer undergoing extended pelvic lymph node dissection: the essential importance of percentage of positive cores. Eur Urol. 2012;61:480-7.

4. D'Amico AV, Chen MH, Renshaw AA, et al. Androgen suppression and radiation vs radiation alone for prostate cancer: a randomized trial. JAMA. 2008;299:289-95.

5. Bakavicius A, Drevinskaite M, Daniunaite $K$, et al. The impact of prostate cancer upgrading and upstaging on biochemical recurrence and cancerspecific survival. Medicina (Kaunas). 2020;56:61

6. Kovac E, Vertosick EA, Sjoberg DD, et al. Effects of pathological upstaging or upgrading on metastasis and cancer-specific mortality in men with clinical low-risk prostate cancer. BJU Int. 2018:122:1003-9.

7. Barentsz JO, Weinreb JC, Verma S, et al. Synopsis of the PI-RADS v2 guidelines for multiparametric prostate magnetic resonance imaging and recommendations for use. Eur Urol. 2016;69:41-9.

8. Varma M, Narahari K, Mason M, et al. Contemporary prostate biopsy reporting: insights from a survey of clinicians' use of pathology data. J Clin Pathol. 2018;71:874-8.

9. Brimo F, Vollmer RT, Corcos J, et al. Prognostic value of various morphometric measurements of tumour extent in prostate needle core tissue. Histopathology. 2008;53:177-83.

10. Bullock N, Simpkin A, Fowler S, et al. Pathological upgrading in prostate cancer treated with surgery in the United Kingdom: trends and risk factors from the British Association of Urological Surgeons Radical Prostatectomy Registry. BMC Urol. 2019;19:94.

11. Budaus $L$, Graefen $M$, Salomon G, et al. The novel nomogram of Gleason sum upgrade: possible application for the eligible criteria of low dose rate brachytherapy. Int J Urol. 2010;17:862-8.
12. Chun FK, Steuber T, Erbersdobler A, et al. Development and internal validation of a nomogram predicting the probability of prostate cancer Gleason sum upgrading between biopsy and radical prostatectomy pathology. Eur Urol. 2006;49:820-6.

13. Epstein Jl, Feng Z, Trock BJ, et al. Upgrading and downgrading of prostate cancer from biopsy to radical prostatectomy: incidence and predictive factors using the modified Gleason grading system and factoring in tertiary grades. Eur Urol. 2012;61:1019-24.

14. Kulkarni GS, Lockwood G, Evans A, et al. Clinical predictors of Gleason score upgrading: implications for patients considering watchful waiting, active surveillance, or brachytherapy. Cancer. 2007;109:2432-8.

15. Kvale R, Moller B, Wahlqvist R, et al. Concordance between Gleason scores of needle biopsies and radical prostatectomy specimens: a populationbased study. BJU Int. 2009;103:1647-54.

16. Moussa AS, Kattan MW, Berglund R, et al. A nomogram for predicting upgrading in patients with low- and intermediate-grade prostate cancer in the era of extended prostate sampling. BJU Int. 2010;105:352-8.

17. Wang JY, Zhu Y, Wang CF, et al. A nomogram to predict Gleason sum upgrading of clinically diagnosed localized prostate cancer among Chinese patients. Chin J Cancer. 2014;33:241-8.

18. Davies JD, Aghazadeh MA, Phillips S, et al. Prostate size as a predictor of Gleason score upgrading in patients with low risk prostate cancer. J Urol. 2011;186:2221-7

19. Gofrit ON, Zorn KC, Taxy JB, et al. Predicting the risk of patients with biopsy Gleason score 6 to harbor a higher grade cancer. J Urol. 2007;178:1925-8.

20. Huo AS, Hossack T, Symons JL, et al. Accuracy of primary systematic template guided transperineal biopsy of the prostate for locating prostate cancer: a comparison with radical prostatectomy specimens. J Urol. 2012;187:2044-9.

21. Truong M, Slezak JA, Lin CP, et al. Development and multi-institutional validation of an upgrading risk tool for Gleason 6 prostate cancer. Cancer. 2013;119:3992-4002.

22. Le JD, Tan N, Shkolyar E, et al. Multifocality and prostate cancer detection by multiparametric magnetic resonance imaging: correlation with whole-mount histopathology. Eur Urol. 2015;67:569-76.

23. Park SY, Jung DC, Oh YT, et al. Prostate cancer: PI-RADS version 2 helps preoperatively predict clinically significant cancers. Radiology. 2016;280:108-16.

24. Song W, Bang SH, Jeon HG, et al. Role of PI-RADS version 2 for prediction of upgrading in biopsy-proven prostate cancer with Gleason score 6. Clin Genitourin Cancer. 2018;16:281-7.

25. Gondo T, Hricak H, Sala E, et al. Multiparametric 3T MRI for the prediction of pathological downgrading after radical prostatectomy in patients with biopsy-proven Gleason score $3+4$ prostate cancer. Eur Radiol. 2014:24:3161-70.

26. Capitanio U, Karakiewicz PI, Valiquette L, et al. Biopsy core number represents one of foremost predictors of clinically significant gleason sum upgrading in patients with low-risk prostate cancer. Urology. 2009;73:1087-91.

27. He B, Chen R, Gao X, et al. Nomograms for predicting Gleason upgrading in a contemporary Chinese cohort receiving radical prostatectomy after extended prostate biopsy: development and internal validation. Oncotarget. 2016;7:17275-85.

\section{Publisher's Note}

Springer Nature remains neutral with regard to jurisdictional claims in published maps and institutional affiliations. 